\title{
Outcomes for proximal femur fractures
}

\author{
Sebastiano Cudoni, Pietro Zedde \\ UOC Ortopedia e Traumatologia, Ospedale Giovanni Paolo II, ASSL Olbia, Italy
}

\begin{abstract}
SUMmARY
Fractures of the proximal femur are among the most frequent lesions seen today on account of the increasing mean age of the population. Mortality related to these fractures in elderly patients is around $10 \%$ at one month, and $20-30 \%$ a year from the fracture. Failures after treatment for medial and lateral fractures of the neck of the femur may be attributed to failed osteosynthesis, failure of the prosthetic implant, or minor complications. To prevent complications, multidisciplinary assessment of the patient is essential, with correct preoperative planning, appropriate selection of the osteosynthesis material or prosthesis, and precise, prompt surgery respecting all tissues.
\end{abstract}

Key words: proximal femur fractures, outcomes

Received: February 20, 2020

Accepted: February 28, 2020

\author{
Correspondence \\ Pietro Zedde \\ UOC Ortopedia e Traumatologia, \\ Ospedale Giovanni Paolo II, ASSL Olbia, \\ via Bazzoni-Sircana, 07026 Olbia, Italy \\ E-mail: pietrozedde@tiscali.it
}

Conflict of interest

The Author declares no conflict of interest

How to cite this article: Cudoni S, Zedde P. Outcomes for proximal femur fractures. Lo Scalpello 2020;34:47-51. https://doi. org/10.36149/0390-5276-007

(C) Ortopedici Traumatologi Ospedalieri d'Italia (O.T.O.D.i.) 2020

\section{(c) (i) () $\Theta$}

This is an open access article distributed in accordance with the CC-BY-NC-ND (Creative Commons Attribution-NonCommercial-NoDerivatives 4.0 International) license. The article can be used by giving appropriate credit and mentioning the license, but only for non-commercial purposes and only in the original version. For further information: https://creativecommons.org/licenses/by-nc-nd/4.0/deed.en

\section{Introduction}

Interest in fractures of the proximal femur is growing today not only on account of their important clinical and economic impact, but also their steadily rising incidence. This mainly reflects the growth of the elderly population and in recent decades has led to exponential increases in the numbers of elderly patients admitted to hospital, with far-reaching socio-economic consequences. It is estimated that by 2050 the number of femoral fractures in the world will reach 63 million, and $60 \%$ of them will be in individuals older than $80^{1}$. Current figures for mortality of elderly patients with femoral fractures are around $10 \%$ at one month ${ }^{2}$ and $20-30 \%$ one year from the fracture ${ }^{3}$.

Fractures of the proximal femur can be classified under two main headings, according to the anatomical region involved: medial or intracapsular, and lateral or extracapsular, with differences in their treatment, prognosis and complications. The classification as intra- or extracapsular is based not only on the site of the lesion, but also its important vascular and biomechanical implications.

Treatment of medial and lateral fractures of the neck of the femur may be needed because of:

- failure of osteosynthesis (aseptic necrosis, pseudoarthrosis, mobilisation of the synthesis material, post-treatment fractures);

- failure of the prosthetic implant (acetabular wear, dislocation, aseptic detachment, infection);

- minor complications (nerve deficit, infection of the surgical wound, deep venous thrombosis, bed sores, leg length discrepancy, post-surgical hematoma).

\section{Outcomes of medial fractures of the neck of the femur}

\section{Failure of osteosynthesis with cannulated screws}

Osteosynthesis of fractures of the proximal femur with cannulated screws is indicated for types I and II fractures according to Garden's classification. This is a relatively 
simple mini-invasive procedure that shortens surgical times and preserves the joint, ensuring torsional stability to stress shielding and bending ${ }^{4}$. For correct synthesis, the screws must be positioned parallel, at the periphery of the neck, in order to permit the fracture to slide and engage secondarily. The threads of the screws must go right down into the fragment of the head, beyond the edge of the fracture. The literature does not agree on how many screws are needed for osteosynthesis, but most authors opt for three, placed parallel, $1 \mathrm{~cm}$ from the joint cartilage of the femoral head.

Compared to other synthesis materials, cannulated screws require the patient to wait longer before putting weight on the hip, even only partially, and they can alter the biomechanics of the abductor muscles of the hip (horizontal shortening).

The proximal femoral vascularisation, and particularly at the head, consists of a terminal circle depending mostly on perforating arterial branches, tributaries of the lateral and medial circumflex femoral arteries. Treatment of medial fractures with cannulated screws may therefore lead to avascular necrosis, delayed consolidation and/or pseudoarthrosis.

Avascular necrosis interruption of the arteries, closely depending on the degree of displacement of the fracture, and the increase in intracapsular pressure; both can cause progressive resorption of subchondral bone with loss of the physiological sphericity of the femoral head (Fig. 1).

Pseudoarthrosis is a consequence of poor mechanical stability of the synthesis, and inadequate vascular supply. Clinically, it manifests as increasing pain in the hip and serial X-rays show initial lack of consolidation of the fracture, displacement, mobilization of the synthesis material and in some cases its breakage ${ }^{5}$.

Mobilization of the screws is related to poor quality of the bone and/or technical error in placement (Fig. 2); it calls for

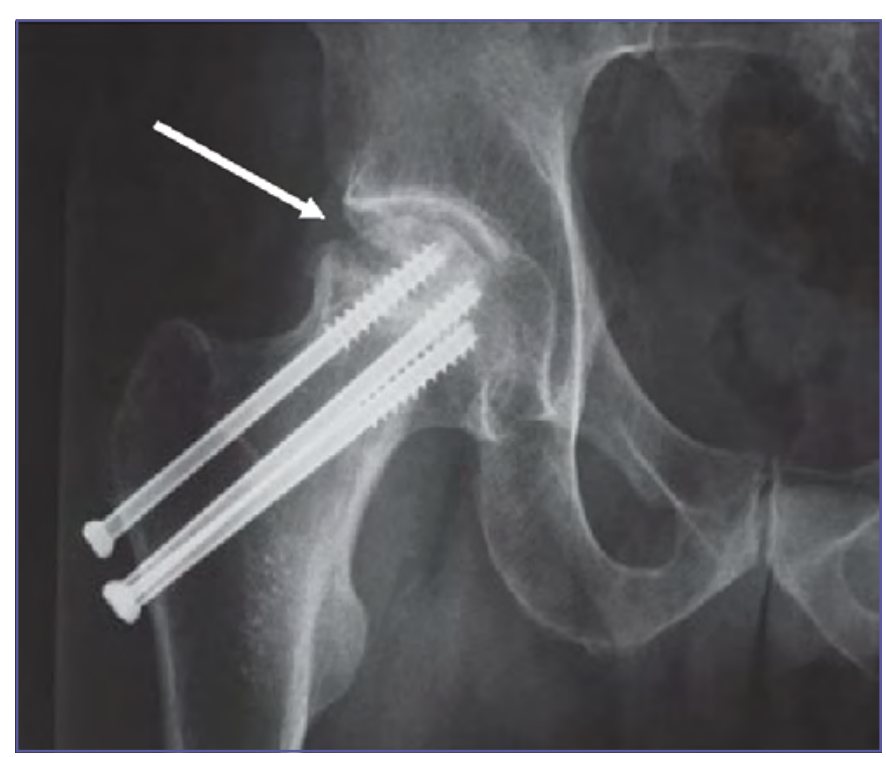

Figure 1. Avascular necrosis after treatment with cannulated screws.

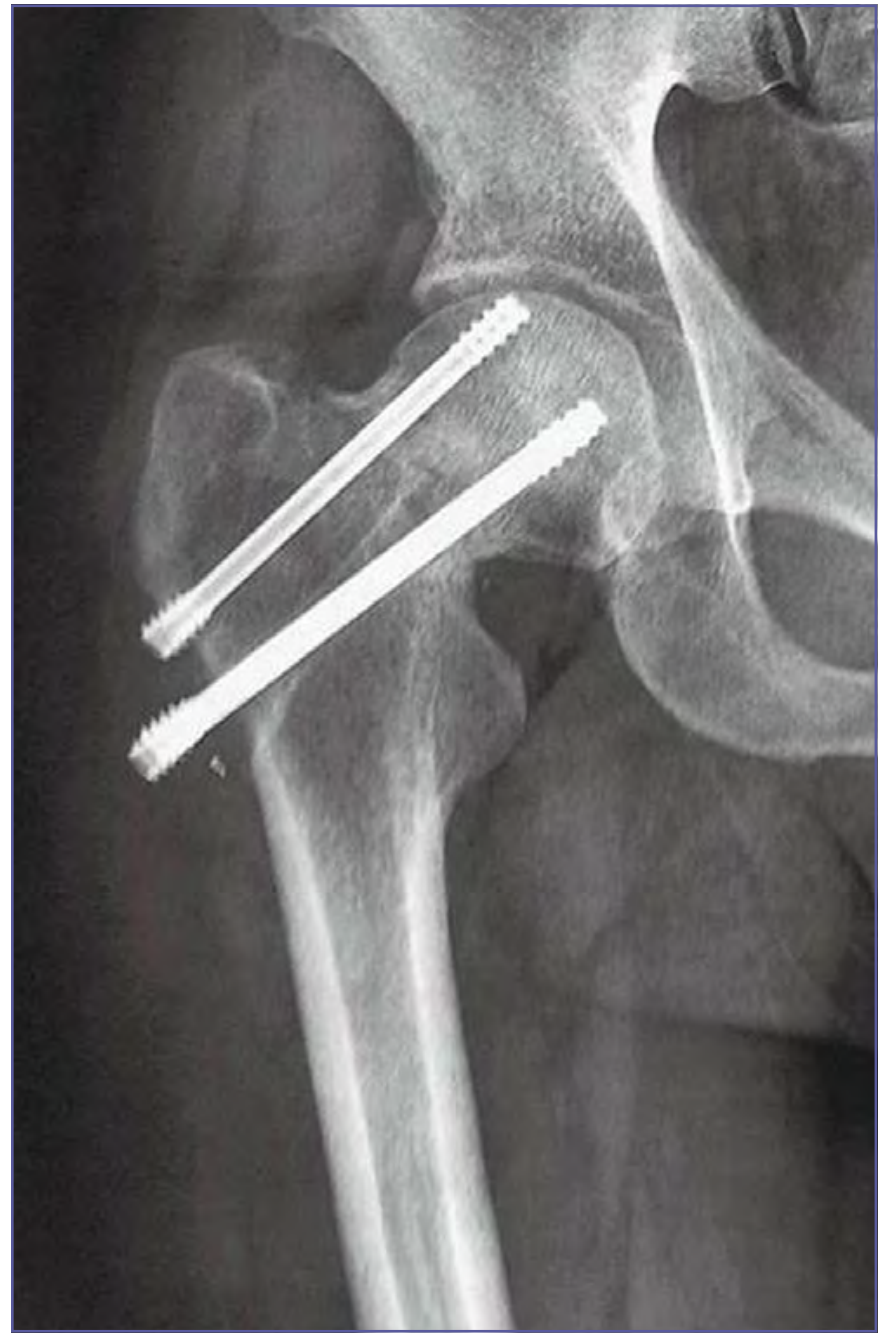

Figure 2. Abnormal synthesis with cannulated screws.

re-operation for total hip replacement. Screws coated with hydroxyapatite are now available, that ensure greater stability of the synthesis even in patients with poor-quality bone ${ }^{6}$.

The incidence of complications is related to the use of cannulated screws, depending on the patient's age. Fractures in young/adult subjects are generally due to high-energy trauma, and present displacement. Slobogean et al., in a meta-analysis in 2015, reported that in these age brackets, after osteosynthesis avascular necrosis was recorded in $14.3 \%$, failure of synthesis in $9.7 \%$ and pseudoarthrosis in $9.3 \%{ }^{7}$. Medial fractures in the elderly - generally compound (Garden I or II) - may present 10-30\% of avascular necrosis, $10-15 \%$ failure of synthesis, and 3-10\% pseudoarthrosis ${ }^{1,4}$.

\section{Failure of the prosthesis}

Various classification systems are available to define medial fractures of the femoral neck: AO, Garden, Pauwels. Garden's 
classification permits the application of a functional diagnostictherapeutic algorithm associated with a good clinical outcome. Selecting the treatment for medial fractures must, however, take account not only of the classification of the lesion, but also the patient's age and any co-morbidities.

Total hip replacement is the preferred strategy for Garden grades III and IV medial fractures

The choice of hemiarthroplasty or total hip replacement must be based on analysis of various factors: the patient's age, symptomatic coxarthrosis or rheumatoid arthritis, the patient's level of activity and neurological status before the fracture. High functional demand should orient the decision towards total replacement.

Hemiarthroplasty involves shorter operating times, rapid functional recovery, and a low rate of dislocation ${ }^{8}$. The rate of revision for complications related to this type of implant ranges from $4-14 \%{ }^{9}$.

Acetabular wear or cotyloiditis is a complication of hemiarthroplasty arising with approximately $0-25 \%$ of hip implants. The patient complains of pain on walking, and X-rays show a narrowing of the articular interline, reaching erosion and protrusion of the acetabular fundus. Cotyloiditis generally calls for revision and total replacement, and some cases require bone grafts. In recent years much attention has focused on 'doublejointed' implants, designed to reduce acetabular erosion and facilitate joint movement. However, these advantages are not borne out by the findings of various studies ${ }^{10}$.

The incidence of periprosthetic hip fractures is constantly rising, with the growing numbers of hip replacements and the mean age of the population. The current figure is from $0.1-3.0 \%$ for first implants, and 6-10\% for revisions ${ }^{11}$. Risk factors are old age, female sex, osteoporosis, displacement of the implant, thinning of the femoral cortices, implantation of an anatomic stem, a cylindrical femur, and instability of the implant (Fig. 3). Joint instability is one complication of hemiarthroplasty and total replacement, and can depend on the patient or the surgical technique. Patient-related risk factors are cognitive decline, neurological dysfunction (spasticity, contraction), muscle disorders, skeletal deformity, and lack of movement on the patient's part ${ }^{4}$. Surgery-related risk factors include failure to restore the offset following erroneous osteotomy or the use of a short-necked prosthesis; reduction of the angle between the center of the cup and edge of the acetabulum, using too small cups, altering the orientation of the stem (antiversion, retroversion).

Zhao et al., in a 2014 meta-analysis, reported a higher rate of revision and fewer dislocations for partial than total hip replacement ${ }^{12}$. The incidence of dislocation arfter hemiarthroplasty ranges between 1.5 and $11 \%{ }^{13}$ with displacement of the implant in $0-9-1.5 \%$. Dislocation of a prosthesis is not traumatic in about $80 \%$ of cases ${ }^{13}$ and occurs mostly in the first three to five months after surgery. For total replacements the orientation of the cotyloid is important, as it is the factor most closely related to instability of the implant.

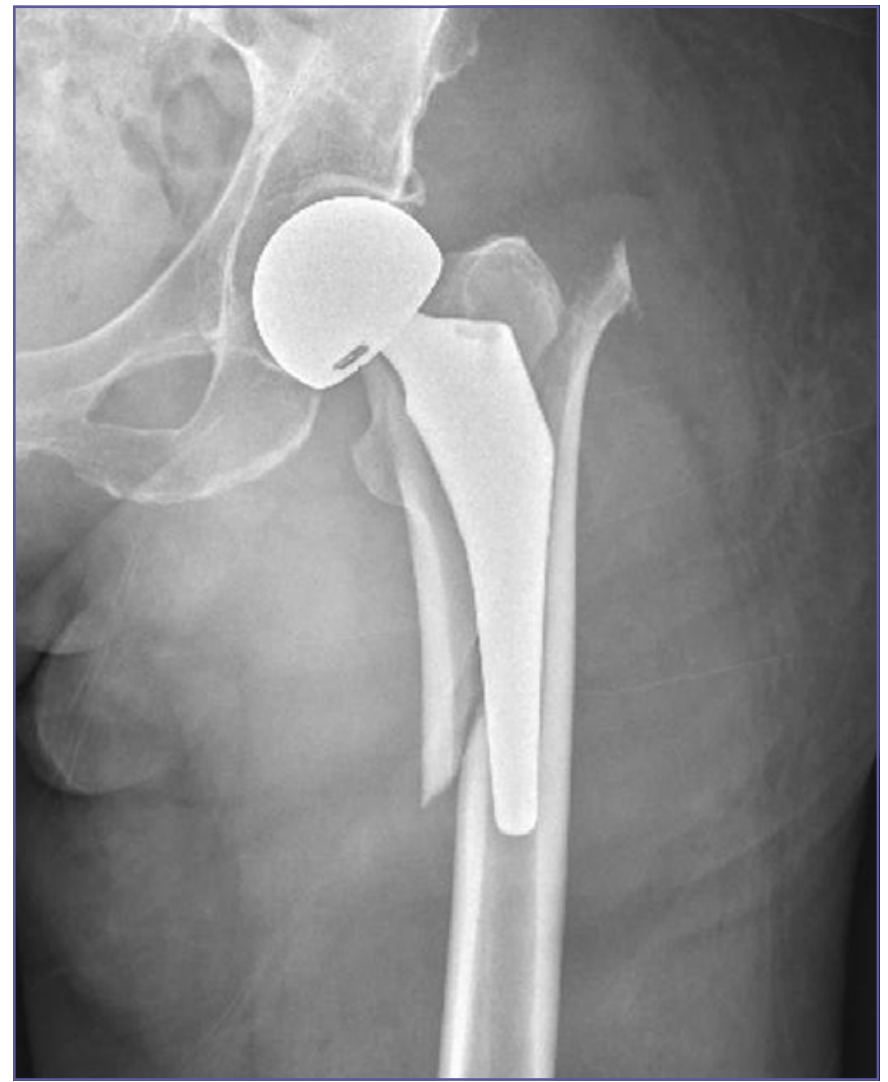

Figure 3. Periprosthetic femur fracture.

Periprosthetic infection is one of the most serious complications, and one of the hardest to treat in modern orthopedic practice. Its incidence is steadily rising, to between $0.5-3.0 \%$ for first replacements, and $20-30 \%$ for revisions. Diagnosing hip replacement infections is complicated because presentation may be aspecific and vary widely, so it is hard to differentiate it from an inflammatory pathology.

The main microrganisms are Staphylococcus aureus and Staphylococcus epidermidis, which cause about $60 \%$ of the infections. These microorganisms form a biofilm on the surface of the prosthesis to boost resistance to the host defences and to antibiotics. Important risk factors include long surgical times, inadequate antibiotic prophylaxis, prolonged surgical drainage or bladder catheter, too many people in the operating room, current or long treatments with cortisone or immune suppressants, obesity, and long hospital stays.

Several algorithms are available to diagnose periprosthetic infection. One of the most widely used currently is that of the Musculoskeletal Infection Society, dated $2011{ }^{14}$. Their algorithm is based on minor and major criteria, and indicates a diagnosis of infection when one of the major criteria is met (two periprosthetic cultures positive for the same microoorganism, or a fistula communicating with the joint), and three of the minor ones (high ESR and CRP, increase in leukocytes in synovial 
fluid, positive leukocyte esterase strip, high percentage of neutrophils in synovial fluid, only one positive periprosthetic culture, positive histology for periprosthetic tissue).

The main changes introduced by the Consensus are abolition of the presence of purulent fluid in the joint, and the introduction of the leukocyte esterase strip. Recently there has been considerable interest in alpha-defensin, a highly sensitive and specific biomarker in synovial fluid ${ }^{15}$.

The increasing presence of low-grade infection, not correctly diagnosed, suggests an explanation for the growing numbers of painful protheses that are are often hard to diagnose properly.

\section{Outcomes of lateral fractures of the femoral neck}

\section{Failure of osteosynthesis with an intramedullary nail}

The treatment of lateral fractures of the proximal femur has undergone considerable changes in the last few decades. From the 1960s, the screw-plate approach was considered the gold standard ${ }^{16-18}$ but more recently, however, in view of the doubtful outcomes of the synthesis material in cases of unstable fractures, intramedullary implants are becoming increasingly widespread ${ }^{19-21}$. Treatments with plate and nail have lost ground. Some recent studies report statistics indicating about $90 \%$ use of nails for intertrochanteric fractures ${ }^{22,23}$. Intramedullary nails offer a low-invasive treatment that ensures stable synthesis and permits immediate weight-bearing and speedy recovery.

With the patient supine the fracture is usually reduced closed, on a traction bed. The good outcome of treatment of lateral fractures depends on mechanically stable osteosynthesis, based on careful planning and the correct use of implants and instruments.

Cut-out is the most common cause of failed osteosynthesis of proximal femoral fractures treated with nails ${ }^{24}$; there is progressive osteolysis of the cancellous, and then of the cortical bone, causing the cephalic synthesis medium to slide at the joint rim (Fig. 4). Cut-out appears to involve various factors, particularly the length of the cephalic screw (TipApex Distance, TAD theory), the parallelism of the screws at the two corticals of the neck in the antero-posterior projection, the instability of the fracture and its non-anatomic reduction. Baumgartner ${ }^{25}$ found TAD was the most reliable factor for predicting cut-out. The TAD is the sum of the distance from the tip of the screw to the center of the femoral head, in both radiographic projections. TAD greater than $25 \mathrm{~mm}$ seems associated with a high incidence of cut-out.

]The Z-effect, caused by the cervico-cephalic screws sliding inversely on each other, is seen with the combination screw and nail because of wrong load distribution on the proximal blocking system (Figs. 5-6).

Pseudoarthrosis of the proximal femur is the cause of 7-20\% of failures after intramedullary nailing; it is generally the result of lack or incomplete reduction of the fracture, with diastasis of fragments or comminution of the medial cortical wall.

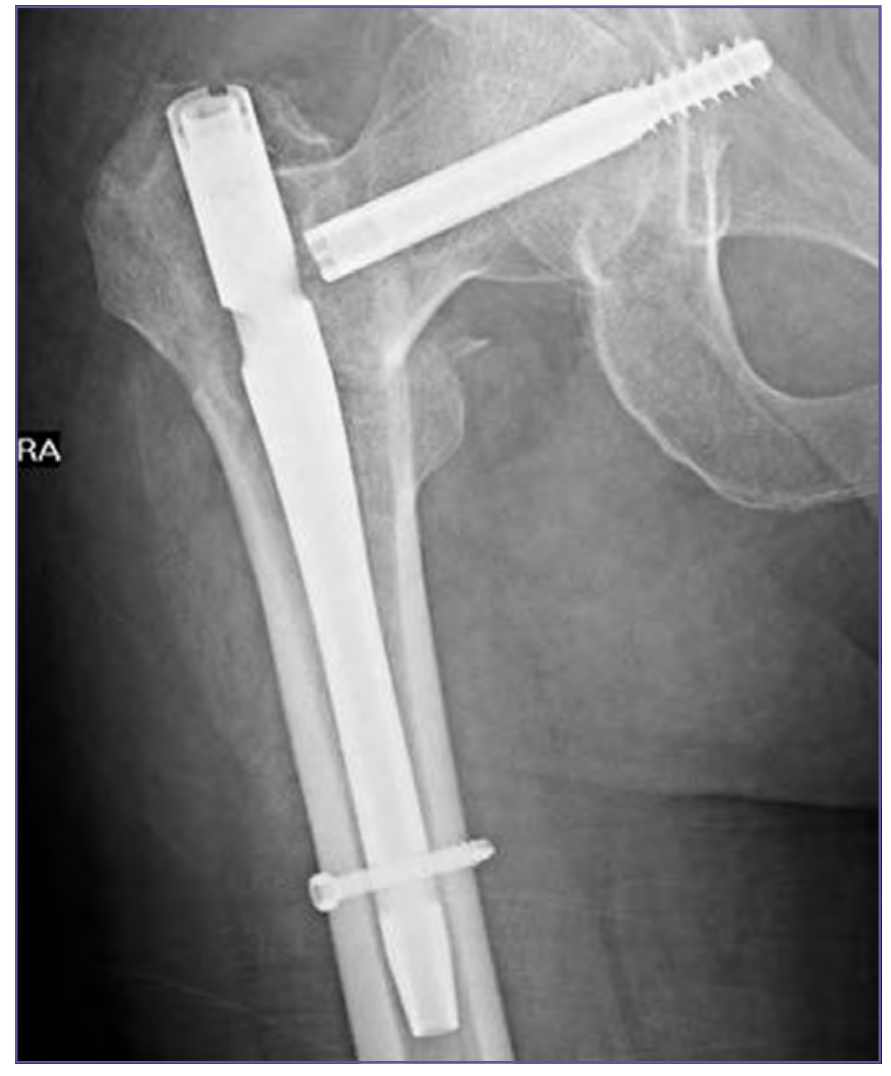

Figure 4. Migration of a cephalic screw.

\section{Conclusions}

The growing practice in hospitals to treat fractures of the neck of the femur within 48 hours in patients over 65 has reduced mortality rates in recent years and achieved worthwhile social and economic

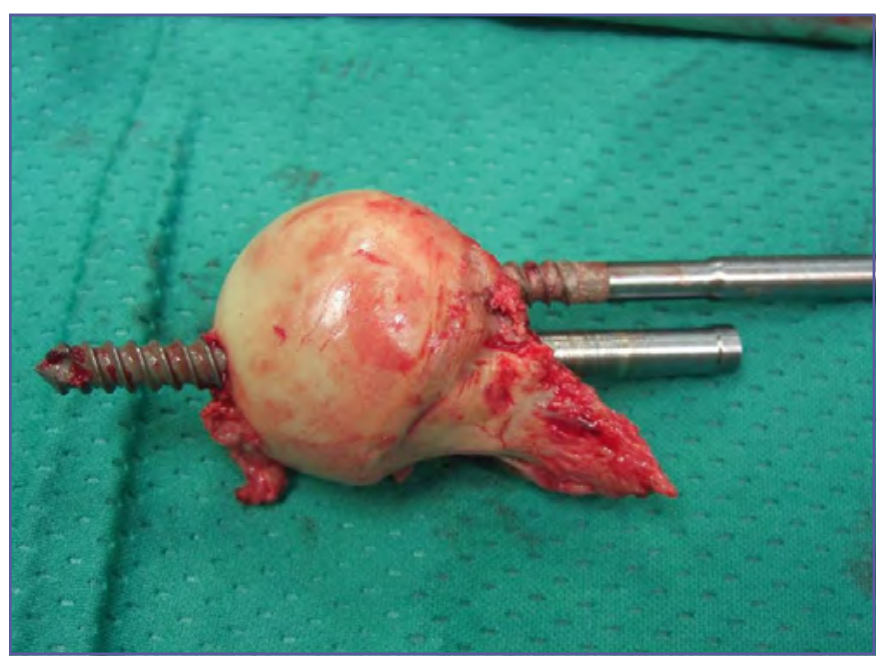

Figure 6. Screws coming out of the femoral head. 


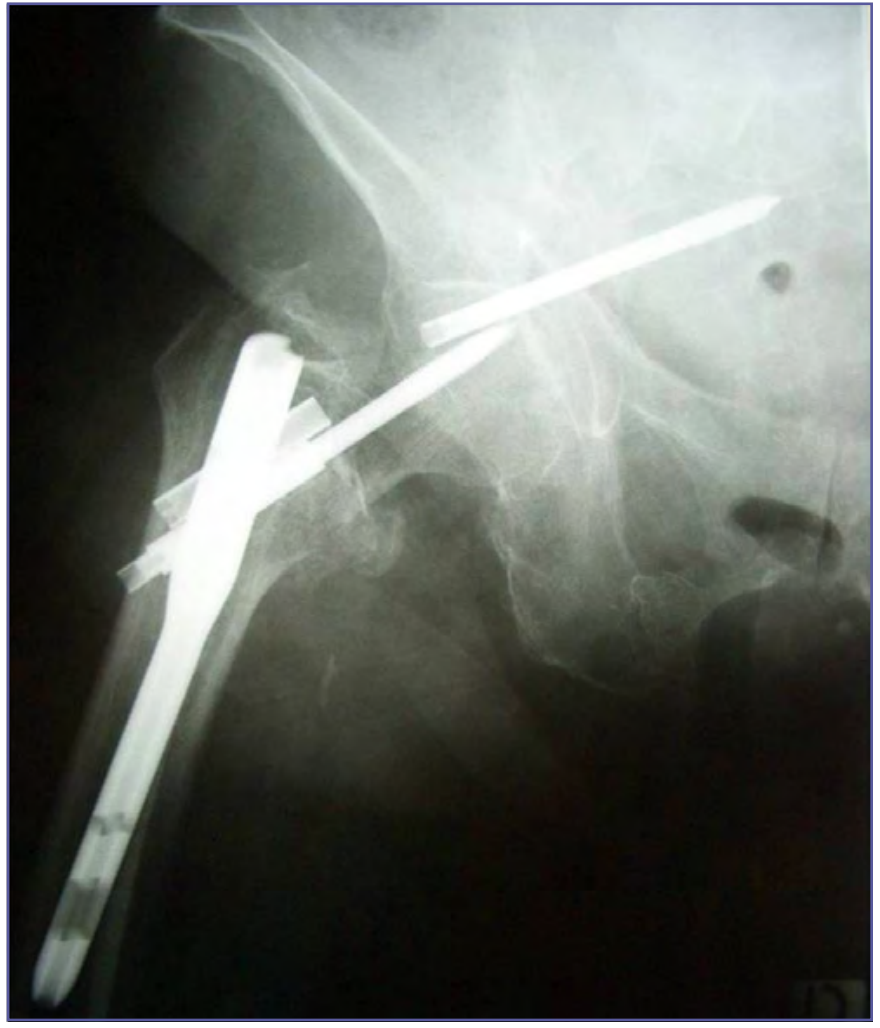

Figure $5 . Z$ effect.

gains. The prevention of pre- and post-operative complications of the fracture calls for a multidisciplinary approach involving orthopedic, geriatric and anesthesia specialists, to take account of the patient's numerous general problems and the type of fracture. A fundamental step is to draw up promptly an overall picture of the patient, correcting any nutritional deficits or electrolyte imbalance to ensure the individuali is stable before surgery. The fracture can then be treated within 48 hours, selecting the most appropriate synthesis material and prosthesis, and employing a speedy, scrupulous surgical technique, respecting all tissues.

\section{References}

Lu Q, Tang G, Zhao X, et al. Hemiarthroplasty versus internal fixation in super-aged patients with undisplaced femoral neck fractures: a 5 -year follow-up of a randomized controlled trial. Arch Orthop Trauma Surg 2017;137:27-35.

2 National Institute for health and clinical excellence. Hip fracture: the management of hip fracture in adults, 2012.

3 Ehlinger M, Moser T, Adam P, et al. Early prediction of femoral head avascular necrosis following neck fracture. Orthop Traumatol Surg Res 2011;97:79-88.

4 Longo F, Bonfiglio S, Salvo GC, et al. Le complicanze delle fratture mediali del collo femorale nel paziente anziano. Giornale Italiano di Ortopedia e Traumatologia 2017;43:117-24.

5 Min BW, Lee KJ, Bae KC, et al. Result of internal fixation for stable femoral neck fractures in elderly patients. Hip pelvis 2016;28:43-8.

6 Faldini C, Moroni A, Grandi G, et al. Trattamento chirurgico delle fratture mediali del collo del femore Garden I-II mediante osteosynthesis con viti percutanee rivestite di idrossiapatite. Giornale Italiano di Ortopedia e Traumatologia 2005;31:247-52.

Slobogean GP, Sprague SA, Scott T, et al. Complications following young femoral neck fractures. Injury 2015;46:484-91.

8 Charnkey J. Arthroplasty of the hip. A new operation. Lancet 1970;1:1129-32.

9 Noailles T, Brulefert K, Chalopin A, et al. What are the risk factors for post-operative infection after hip hemiarthroplasty - Systematic review of literature. Int Orthop 2016;40:1843-8.

10 Zhou Z, Yan F, Sha W, et al. Unipolar versus bipolar hemiarthroplasty for displaced femoral neck fractures in elderly patients. Orthopedics 2015;38:697-702.

11 Kouyoumdjian P, Dhenin A, Dupeyron A, et al. Periprosthetic fracture in the elderly with anatomic modular cementless hemiarthroplasty. Orthop Traumatol Surg Res 2016;102:701-5.

12 Zhao Y, Fu D, Chen K, et al. Outcome of hemiarthroplasty and total hip replacement for active elderly patients with displaced femoral neck fractures: a meta-analysis of 8 randomized clinical trials. Plos One 2014:e 98071.

13 Li L, Ren J, Liu J, et al. What are risk factors for dislocation of hip bipolar hemiarthroplasty through the anterolateral approach? A nested case-control study. Clin Orthop Relat Res 2016;474:2622-9.

14 Parvizi J, Zmistowski B, Berbari EF, et al. New definition for periprosthetic joint infection: from the workgroup of the musculoskeletal infection society. Clin Orthop Related Res 2011;469:2992-4.

15 Bonazinga T, Tanzi P, Zahar A, et al. Il ruolo dell'alfa-defensina nella diagnosi delle infezioni peri-protesiche - una revisione della letteratura. Giornale Italiano di Ortopedia e Traumatologia 2017;43:172-5.

16 Andruszkow H, Frink M, Frömke C, et al. Tip apex distance, hip screw placement, and neck shaft angle as potential risk factors for cut-out failure of hip screws after surgical treatment of intertrochanteric fractures. Int Orthop 2012;36:2347-54.

17 Chirodian N, Arch B, Parker MJ. Sliding hip screw fixation of trochanteric hip fractures: outcome of 1024 procedures. Injury 2005;36:793-800.

18 Güven M, Yavuz U, Kadioğlu B, et al. Importance of screw position in intertrochanteric femoral fractures treated by dynamic hip screw. Orthop Traumatol Surg Res 2010;96:21-7.

19 Barton TM, Gleeson R, Topliss C, et al. A comparison of the long gamma nail with the sliding hip screw for the treatment of AO/OTA 31-A2 fractures of the proximal part of the femur: a prospective randomized trial. J Bone Joint Surg Am 2010;92:792-8.

20 Al-yassari G, Langstaff RJ, Jones JW, et al. The AO/ASIF proximal femoral nail (PFN) for the treatment of unstable trochanteric femoral fracture. Injury 2002;33:395-9.

21 Bojan AJ, Beimel C, Speitling A, et al. Consecutive gamma nails. 12 Years experience at a single centre. BMC Musculoskelet Disord 2010;26;11:133.

22 Lee YK, Yoon BH, Nho JH, et al. National trends of surgical treatment for intertrochanteric fractures in Korea. J Korean Med Sci 2013;28:1407-8.

23 Page PR, Lord R, Jawad A, et al. Changing trends in the management of intertrochanteric hip fractures - a single centre experience. Injury 2016;47:1525-9.

24 Leonardi M, Clatti R, Zammiti A, et al. Le fratture laterali del collo femore dell'anziano: nuova metodica mini-invasiva a basso rischio di cut-out. Giornale Italiano di Ortopedia e Traumatologia 2013;39:11-5.

25 Baumgaertner MR, Curtin SL, Lindskog DM, et al. The value of the tip-apex distance in predicting failure of fixation of peritrochanteric fractures of the hip. J Bone Joint Surg Am 1995;77:1058-64.

26 Nikura T, Lee SY, Sakai T, et al. Nonunion with breakage of gamma nail and subsequent fracture in the ipsilateral femur. Case Rep Med 2013;3:534570. 